$\xi=-1$

\title{
Classification of Hyperspectral Remote Sensing for Production Minerals Mapping Using Geological Map and Geomatics Techniques
}

\author{
Hussein Sabah Jaber ${ }^{1 *}$ \\ ${ }^{1}$ Department of Surveying Engineering, University of Baghdad \\ *E-mail: husseinsabah00@gmail.com
}

\begin{abstract}
The classification of hyperspectral images is an interesting job since the data dimension is huge for conventional classification procedures; normally several hundreds of spectral bands are attained for each image. These spectral bands can supported very rich spectral data of each pixel to find objects material .The objective of this research is to classify hyperspectral images for detection and production of detailed minerals mapping using geological map and Environment for Visualizing Images (ENVI) software. In this research, ASTER data and geological map have been used. Some techniques on these data are used such as enhancement, matching (linking), Decorrelation, Band Ratio, stacking image and classification. The results showed that comparison of the two classification results showed the classification of stack image with the aspect and the slope provide more information than classification of ASTER image alone. Also, using ENVI software to generate 3D surface views.It concluded that capability of hyperspectral and its differentiation with multispectral data to extract detailed features from ASTER image.
\end{abstract}

Keywords: Band ratio, Classification, De-correlation, Hyperspectral Stacking.

\section{Introduction}

Hyperspectral remote sensors gather image data instantaneously in dozens or hundreds of adjacent spectral, narrow bands. These amounts make it potential to develop a continuous spectrum for each image cell [1]. Hyperspectral images comprise a rich of data, but interpreting them needs a sympathetic of exactly what ground materials properties that can try to measure, and how relate to the measurements essentially made by the hyperspectral sensor. Imaging spectrometers are instruments which used for producing hyperspectral images [2]. The ratio of reflected energy to incident energy as a function of wavelength is called spectral reflectance. Since energy at certain wavelengths is scattered or absorbed to different degrees, reflectance differs with wavelength for most materials [3].When compare spectral reflectance curves (plots of reflectance versus wavelength) for different materials, these reflectance variations are evident [4]. Also, the spectral information alone does not permit the separation of structures. For example, the roofs of the roads and some buildings can be made via the similar material (asphalt). Hence, geometrical features and contextual data are crucial for hyperspectral images classification [5].

[6] Studied the classification of a hyperspectral image sequence quantities to find which pixels comprise different spectrally distinctive materials that have been identified via the user. Some methods for classification of multi/hyperspectral pixels have been used from maximum likelihood and minimum distance classifiers to correlation/ matched filter-based methods such as spectral angle mapper and spectral signature matching. [7] Defined unsupervised image classification as documentation of natural groups within multispectral data. In unsupervised image classification does not use training data for individual information classes as the basis for classification; image pixels are examined. Supervised image classification defines information categories and then examines their spectral separately [8].

The aim of this research is to classify hyperspectral images for detection and production of detailed minerals mapping using geological map and geomatics techniques such as ENVI software.

\section{Data used and materials}

ASTER data which is the Advanced Space borne Thermal Emission and Reflection Radiometer is used to produce detailed maps of land surface temperature, reflectance, and elevation for the study area in Malaysia. The coordinated system of EOS satellites, including Terra, is a major component of NASA's Science Mission Directorate and the Earth Science Division [9]. The data set provided consist of a subset of an ASTER scene (band 1 to14 covering the VNIR-SWIR-TIR) and a digital elevation model as shown in table 1 [10]. The DEM has been generated using the stereo capability of ASTER from the $3 \mathrm{~B}$ and $3 \mathrm{~N}$ visible bands.

Table 1: ASTER Data.

\begin{tabular}{cccccc}
\hline Satellite & Sensor & Bands & $\begin{array}{c}\text { Spectral Range } \\
\boldsymbol{\mu m}\end{array}$ & $\begin{array}{c}\text { Scene Size } \\
\mathbf{k m}\end{array}$ & $\begin{array}{c}\text { Pixel Res } \\
\mathbf{m}\end{array}$ \\
\hline VNIR & $1-3$ & $0.520-0.860$ & $120 \times 150$ & 15 \\
\hline
\end{tabular}

Copyright $\odot 2018$ Authors. This is an open access article distributed under the Creative Commons Attribution License, which permits unrestricted use, distribution, and reproduction in any medium, provided the original work is properly cited. 


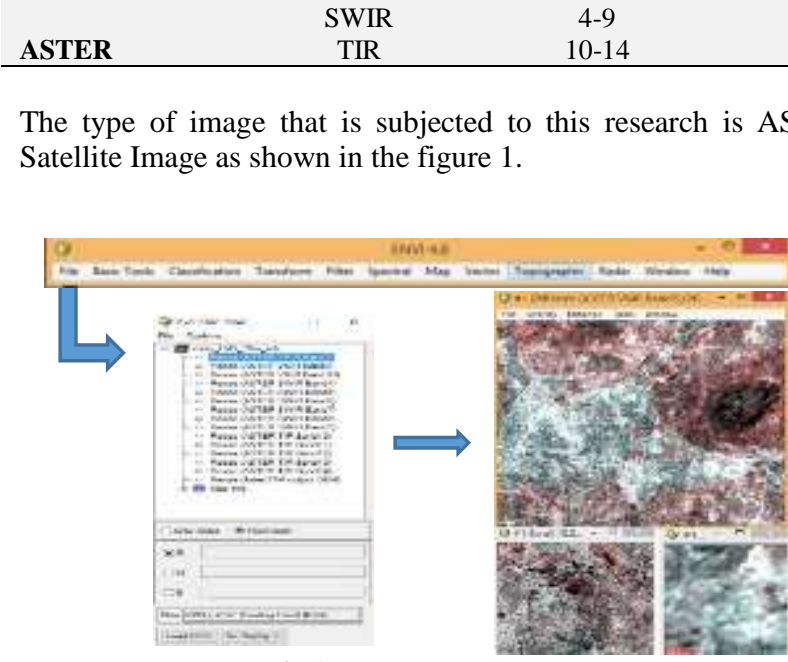

Fig.1: ASTER Satellite Image.

Also, the geological map displayed as in ENVI software as shown in the figure 2. Also, displaying the legend using ENVI software is important because of a better understanding of the location of the minerals across the geological map as shown in the figure 3 .

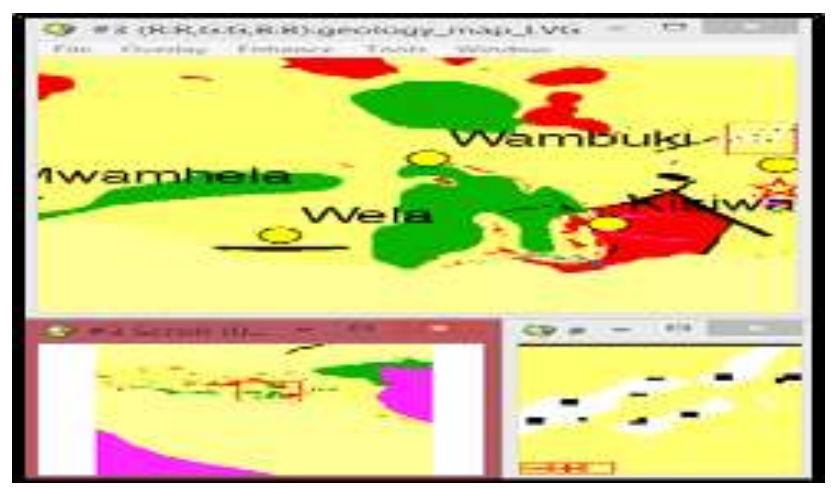

Fig.2: geological map.

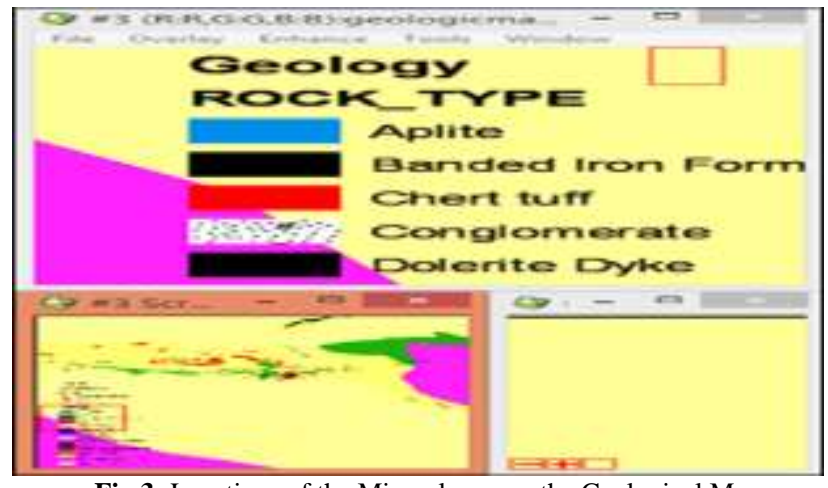

Fig.3: Locations of the Minerals across the Geological Map.

\section{Methodology}

There are several procedures of this research as following:

\subsection{Enhancement process of the ASTER image:}

Image enhancement objective is to supply'enhanced' input for other automated image processing techniques, or to develop the interpretation of data in images for human viewers[11]. So, in ENVI software, there are various types of enhancement algorithms. In this research, an area of interest in the image was selected the darker areas (blobs) in the ASTER scene to enhance specific features in the image. Two different algorithms were selected using ENVI software to see which one is better for visualization. These algorithms include: Square roots and Gaussian were selected
$1.600-2.430$

$8.125-11.65$ because these algorithms are commonly used in ENVI. From two enhancement algorithms which were applied to get clear visualization, the Gaussian algorithm is a better visualization as shown in the figure 4.

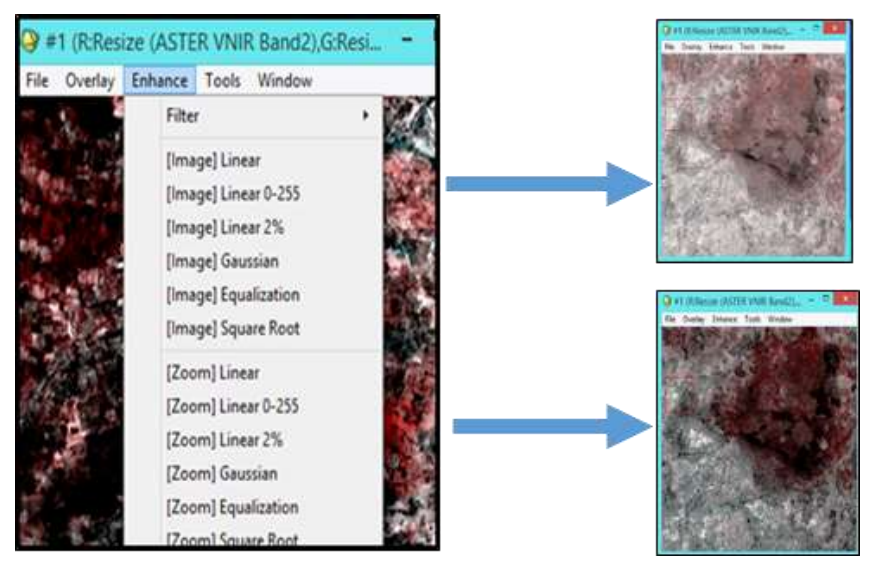

Fig4: Enhancement of ASTER Satellite Image

\subsection{Matching (linking) process:}

Figure 5 showed the Geology map_ (right) and the Image (Left) of the study area before matching (linking) between them. Whereas the figure 6 showed the matching between Geology map (right) with the Image (Left) using ENVI software.

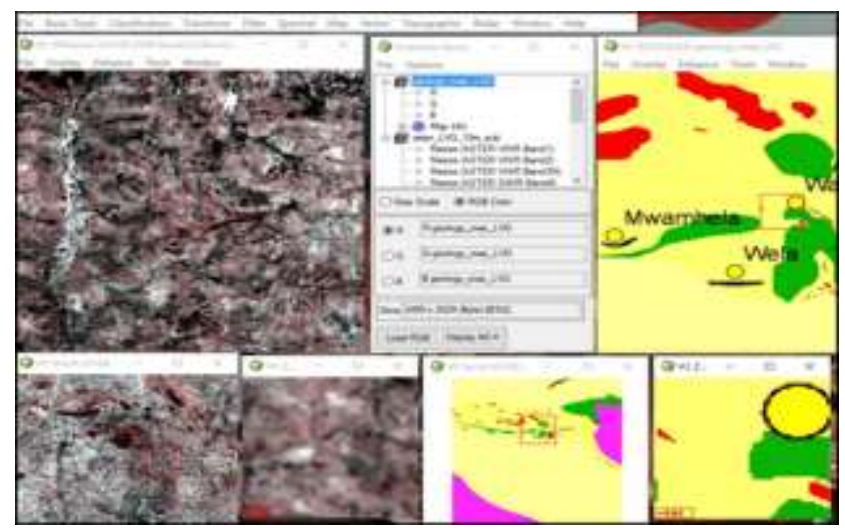

Fig. 5: Geology Map (Right) and the ASTER Image (Left) before Matching (Linking).

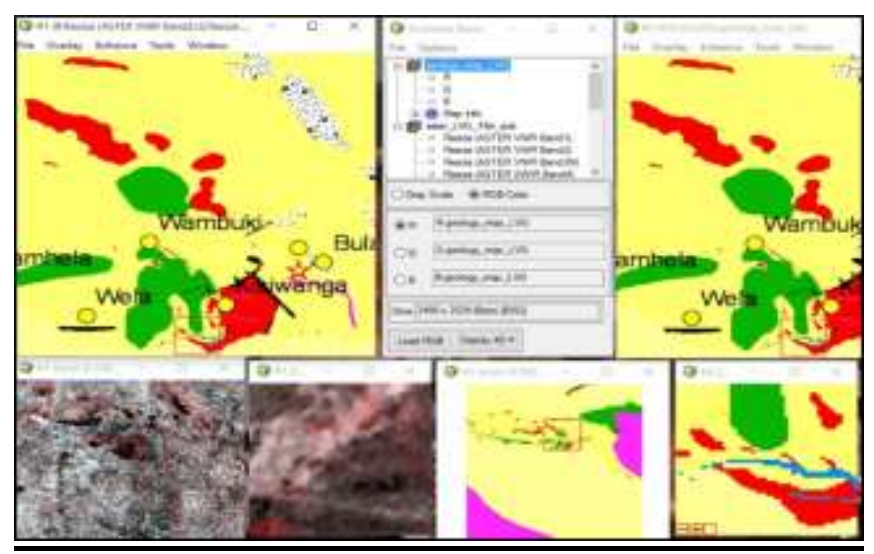

Fig. 6: Geology Map (Right) and the ASTER Image (Left) after Matching (Linking).

\subsection{De-correlation technique:-}

De-correlation Stretch is used to eliminate the high correlation usually created in multispectral information collections and to 
yield a more colorful multiple image [12]. The highly correlated information collections frequently create quite bland color images. De-correlation technique needs three bands for input. These bands must be stretched byte information or can be nominated from an open color display as shown in the figure 7 .

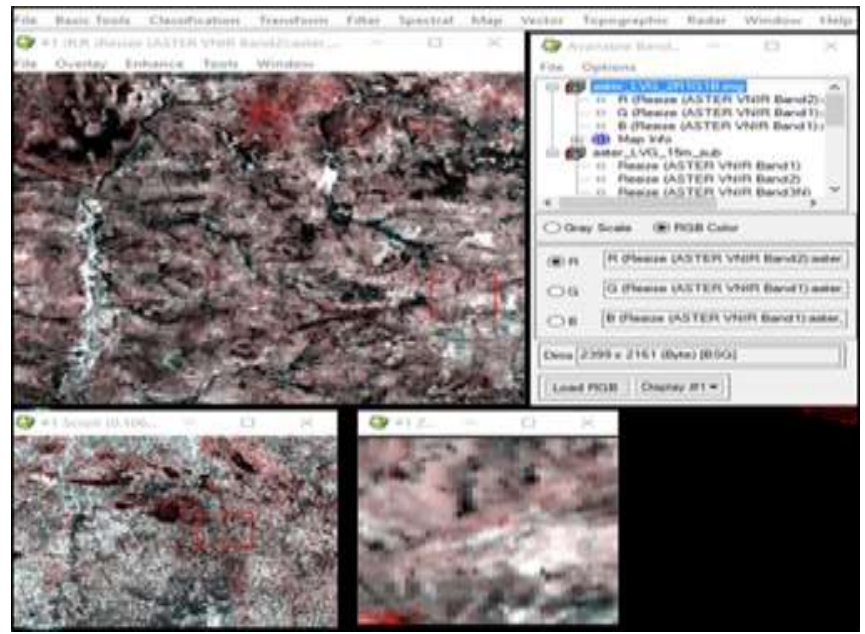

Fig.7: De-correlation Stretch of the Image.

\subsection{Band ratio technique:-}

Band ratio is used to analysis multispectral images to detect feature that can be enhanced in particular range of wavelength, below image shows the band ration of 4 and 6 from the used aster data [13] .Other rations can be investigated to detect different feature after analyzing the spectral signature if known classes.

Ratio images allow to calculate spectral slopes that often can be interpreted as indicative for clay mineral abundance, iron oxides etc. Another (advantage or disadvantage) feature of ratio images is that the albedo and topographic effects are rationed out.

Calculate the ratio using ENVI software as shown in the figure 8.

ASTER band 3/band 1 for iron oxides

ASTER band 4/band 6 for clay minerals

ASTER band 8/band 9 for carbonate/sulphate minerals
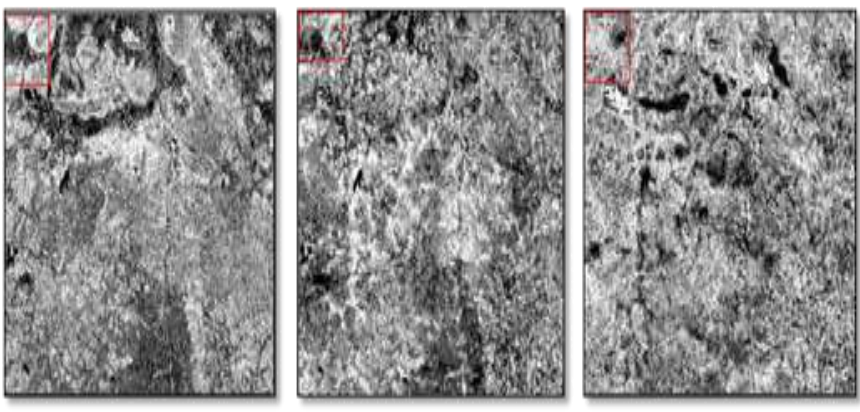

Fig.8: Band Ratio Technique of the Image

Compare the ratio images individually. You may also create a false color composite image by combining the above ratio images as RGB image. Try to reason how to interpret the image colors. The ratio of two bands eliminates considerably of the influence of clarification in spectral changes analysis. This is likely since the composition changes between two geologic units (in this photo limestone, which is "grey white" in RGB321 and "red bed" which is red in RGB321) find the ratio of any two bands, while the clarification finds amount of $\mathrm{DN}$ received via the satellite.

\subsection{Creating the topographic and stacking layer:}

The topographic layer can be created using the DEM. The purpose of using topographic modeling is to create $3 \mathrm{D}$ surfaces and derive other data such as aspect, convexities, and slope.
The topographic layer enables to uncover various features and add them to the existing original layer to provide more information in the classification.

In this research, building of the stacking was performed only with the aspect and the slope. The stack image has provided the image as shown in the figure 9. The first image has 14 bands, but after adding the Aspect and another image is generated. This new image is going to be classified for the purpose of comparison to see if there are changes.

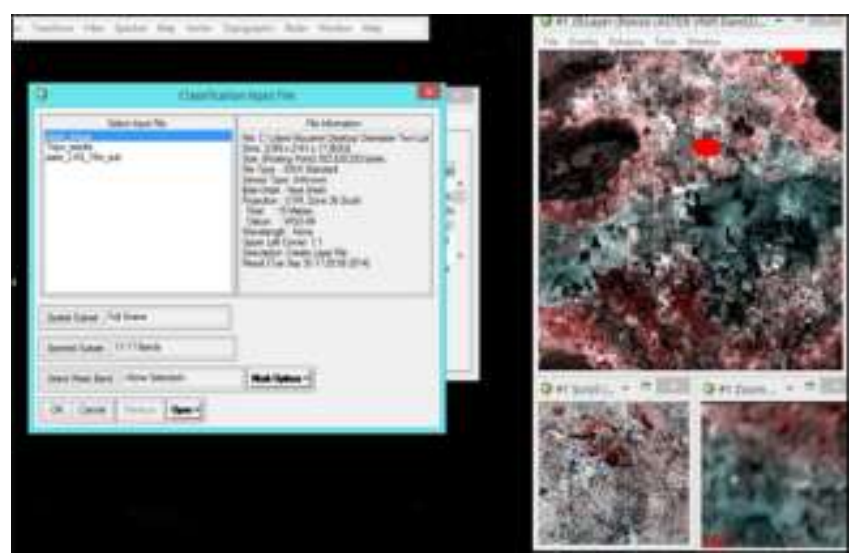

Fig.9: Stack Image of the Study Area

\subsection{Classification process:-}

The aim of image classification is to find and interpret as a distinctive gray level (or color) the features existing in an image in terms of the object [14]. The classification can be unsupervised using field data from Spectroradiometer or be supervised in this case where a satellite image is used [15].

In this research, Region of Interest (ROI) was selected as training sites of the image had four types as following:

Lower Nyanzian, Conglomerate, Chert Tuff and Granite. Figure 10 showed the Region of Interest (ROI).

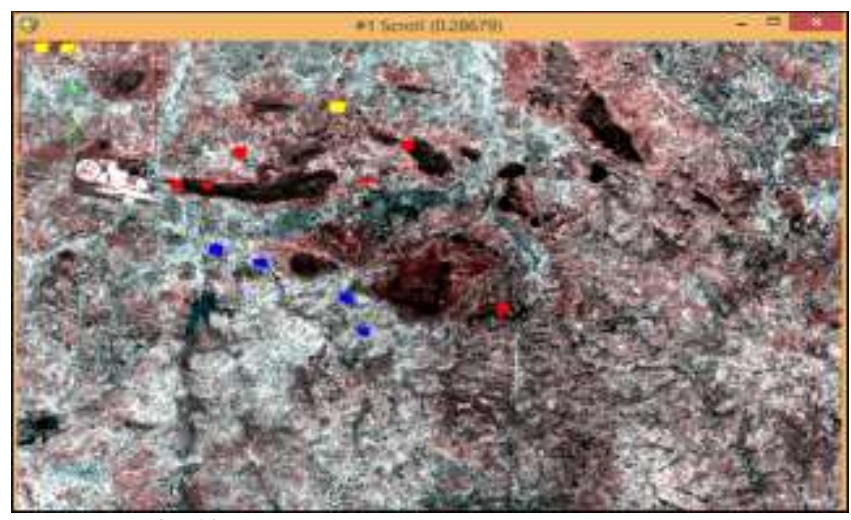

Fig. 10: The Region of Interest (ROI) on the Image

In the classification, there is overlapping of spectral signatures, so a statistical assessment condition to support in the classification of overlying of signatures called (Maximum Likelihood classification) is employed in this research. Also, classification of stack image has been done.

\section{Results and discussion}

After classification of ASTER image using Maximum likelihood method to produce ASTER map of the Region of Interest as shown in the figure 11 where only 14 bands are presented. The $15^{\text {th }}$ band is the DEM (Digital Elevation Model) which is created from terrain elevation data as shown in the figure 12 


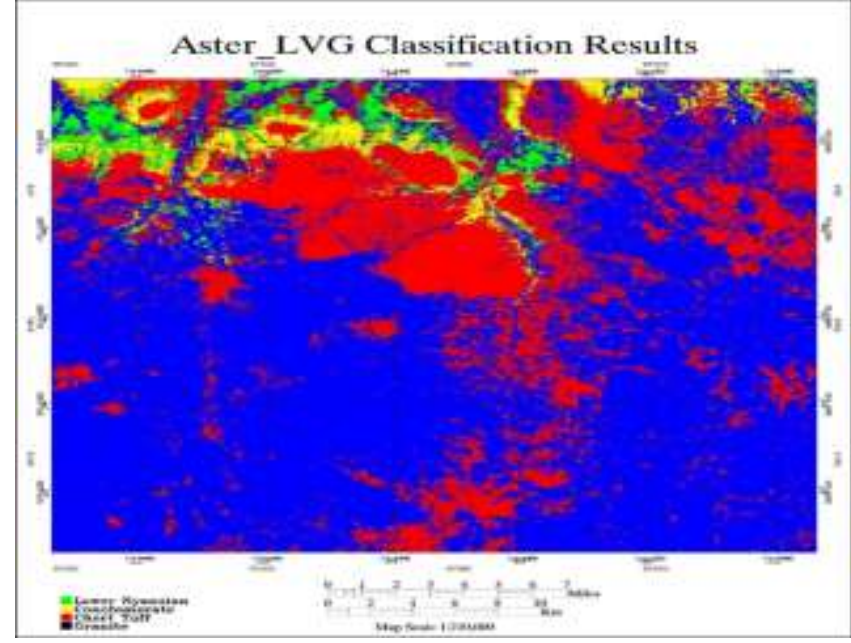

Fig.11: ASTER Classification Map of four Region of Interest.

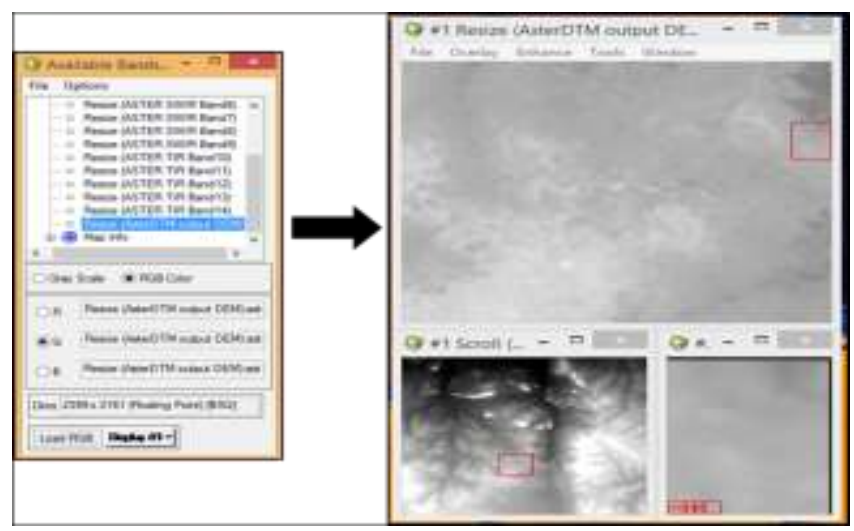

Fig.12: DEM of Study Area.

The result of classification stack image with the aspect and the slope in order to produce a new map and provide more information as shown in the figure 13.

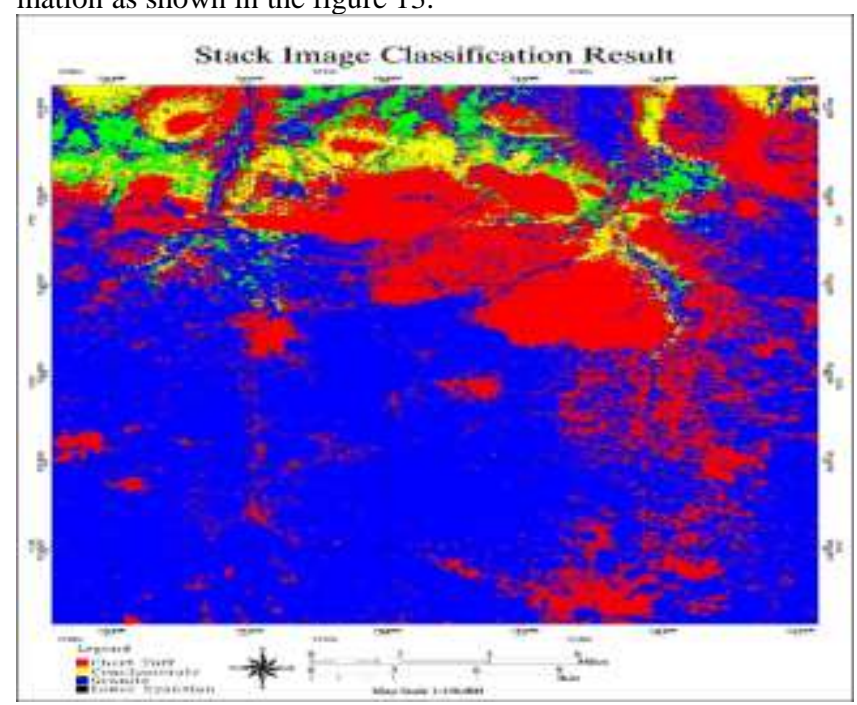

Fig.13: Stack Image Classification of the Region of Interest.

Comparison of the two classification results showed the classification of stack image with the aspect and the slope provide more information than classification of ASTER image as shown in the figure 14 .

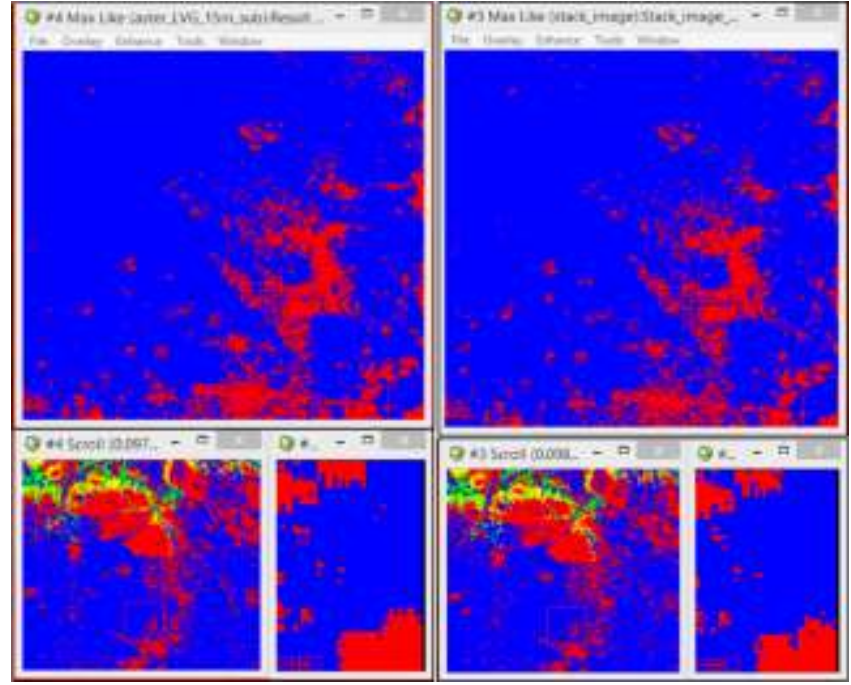

Fig.14: Comparison of the Two Classification Results.

Also, using ENVI software to generate 3D surface views of the study area from DEM as shown in the figure 15.

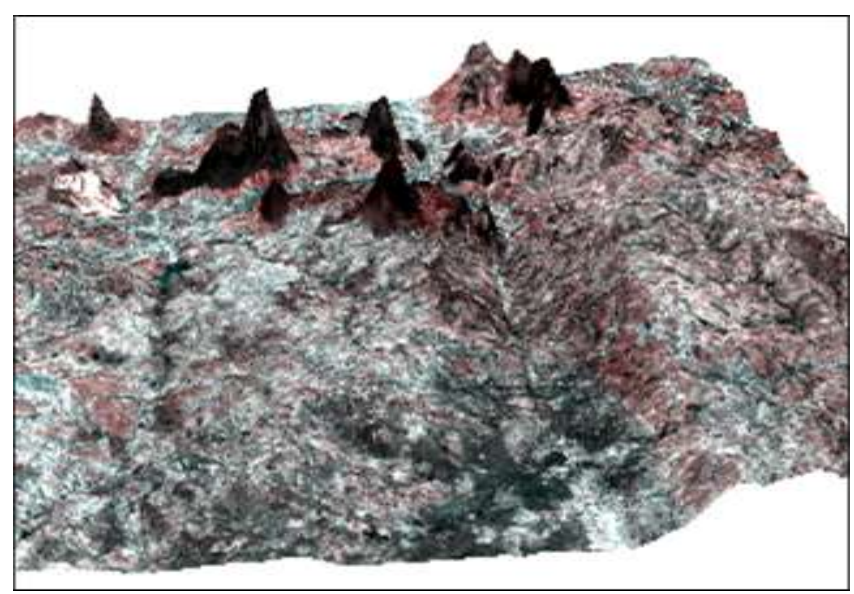

Fig.15: 3D Surface Views of the Study Area.

\section{Conclusion}

With a geological map geocoded, it can determine or detection materials on the image. Moreover, if a sample of spectral profile for each material is an available then it can make this research easier because it can choose that material from all surface of image by using ENVI software. DEM image classification is more accurate than origin image classification that has 15 bands because of DEM image have slope and aspect information. Also, using ENVI software to generate 3D surface views of the study area from DEM. In addition, it concluded that capability of hyperspectral and its differentiation with multispectral data to extract detailed features from ASTER image.

\section{References}

[1] Lattin, J. M., Douglas, J., \& Green, P. E. 2003. Analyzing multivariate data. china:Machine Press, pp. 38-40.

[2] Hardin, P, 2013, Hyperspectral Remote Sensing of Urban Areas.GeographyCompass,7:7-21.doi:10.1111/gec3.12017.

[3] Fang, X., Zhu, X., Wang, Z., Zhao, G., Jiang, Y., \& Wang, Y. A. 2014. Hyperspectral Characteristics of Apple Leaves Based on Different Disease Stress. Remote Sensing Science.

[4] Graham, R.L., 1972. An efficient algorithm for determining the convex hull of a finite planar set," Information Processing Letters, 1 , $132-133$.

[5] Kennedy RE, Townsend PA, Gross JE, Cohen WB, Bolstad P, et al. 2009.Remote sensing change detection tools for natural resource managers: Understanding concepts and tradeoffs in the design of 
landscape monitoring. Projects. Remote sensing of environment, 113: 1382-1396.

[6] Delwiche, S.R., Kim, M.S., 2000. Hyperspectral imaging for detection of scab in wheat. In:Proceedings of SPIE 4203, Biological Quality and Precision Agriculture II, 13-20.

[7] Delwiche, S.R., 2003. Classification of scab and other molddamaged wheat kernels by nearinfrared reflectance spectroscopy. Transactions of the ASAE, 46(3), 731-738.

[8] Bock, C.H., Poole, G.H., Parker, P.E., Gottwald, T.R., 2010. Plant disease severity estimated visually, by digital photography and image analysis, and by hyperspectral imaging. Critical Reviews in Plant Sciences, 29(2), 59-107.

[9] Michael, A., Bhaskar, R. ASTER Users Handbook.Version 2. 2015.

[10] USGS, Japan ASTER Program, 2003, ASTER scene AST_L1B_003_06262000100635, 1B, USGS, Sioux Falls, 6/26/2001.

[11] Nutter, F.W., Gleason, M.L., Jenco, J.H., Christians, N.C., 1993. Assessing the accuracy, intrarater repeatability, and inter-rater reliability of disease assessment systems. Phytopathology, 83, 806-812.

[12] Naidu, R.A, Perry E.M, Pierce FJ, Mekuria, T., 2009. The potential of spectral reflectance technique for the detection of Grapevine leafroll-associated virus-3in two red-berried wine grape cultivars. Computers and Electronics in Agriculture,p: 38-45.

[13] Mahlein A.-K. Rumpf T., Welke, P., Dehne H.-W., Plumer L.,Steiner U., Oreke E.-C. 2013. Development of spectral indices for detecting and identifying plant diseases, Remote Sensing of Environment, 128 (21) pp. 21-30.

[14] Lu D, Mausel P, Brondizio E, Moran E, 2004. Change detection techniques.International journal of remote sensing, 25: 2365-2401.

[15] Lillesand, T.M. and Kiefer, R.W., 1994.Remote Sensing and Image Interpretation, 3rd Ed., John Wiley and Sons, Inc.: Toronto. 